\title{
Elastodynamics for Non-linear Seismic Wave Motion in Real-Time Expert Seismology
}

\author{
Evangelos G. Ladopoulos \\ Interpaper Research Organization, 8, Dimaki Str., Athens, GR - 106 72, Greece
}

(Received 15 May 2012; provisionally accepted 9 February 2013; accepted 19 May 2013)

Based on the new theory of Real-Time Expert Seismology, a non-linear 3-D elastic wave real-time expert system is used for the exploration globa on-shore and off-shore petroleum and gas reserves. This highly innovative and ground-breaking technology uses elastic (seismic) waves moving in an unbounded subsurface medium to searching the on-shore and off-shore hydrocarbon reserves developed on the continental crust and in deeper water ranging from 300 to $3000+\mathrm{m}$. This modern technology can be used in any depth of sea, in oceans all over the world, and for any depth in the subsurface of existing oil and gas reserves.

Furthermore, the various mechanical properties of the rock regulating the wave propagation phenomenon appear as spatially-varying coefficients in a system of time-dependent hyperbolic partial differential equations. The propagation of the seismic waves through the earth subsurface is described by the wave equation, which is finally reduced to a Helmholz differential equation. Then the Helmholtz differential equation is numerically evaluated by using the Singular Integral Operators Method (SIOM). Also, several properties are analysed and investigated for the wave equation.

Finally, an application is proposed for the determination of the seismic field radiated from a pulsating sphere into an infinite homogeneous medium. The acoustic pressure radiated from the above pulsating sphere is determined by the SIOM.

\section{INTRODUCTION}

The new technology Non-Linear Real-Time Expert Seismology is the main and best tool which can be used by the oil and gas industry to map petroleum deposits in the Earth's upper crust. Environmental and civil engineers can also use variants of the above modern technique to locate bedrock, aquifers, and other near-surface features. Academic geophysicists can extend it into a tool for imaging the lower crust and mantle. This method was proposed and investigated by Ladopoulos ${ }^{26-31}$ as an extension of his methods on non-linear singular integral equations in fluid mechanics, potential flows, structural analysis, solid mechanics, hydraulics, and aerodynamics. ${ }^{16-25}$

Seismic wave propagation is the physical phenomenon underlying the Non-Linear Real-Time Expert Seismology, as well as other types of seismology. It is modelled with reasonable accuracy as small-amplitude displacement of a continuum, using various specializations and generalizations of linear elastodynamics. In these models, the various mechanical properties of rock regulating the wave propagation phenomenon appear as spatially-varying coefficients in a system of time-dependent, hyperbolic, partial differential equations.

The Non-Linear Real-Time Expert Seismology seeks to extract maps of the Earth's sedimentary crust from transient, near-surface recordings of echoes stimulated by explosions or other controlled sound sources positioned near the surface. Reasonably accurate models of seismic energy propagation take the form of hyperbolic systems of partial differential equations, in which the coefficients represent the spatial distribution of various mechanical characteristics of rock, like density, stiffness, etc. The exploration geophysics community has developed various methods for estimating the Earth's structure from seismic data; however the modern technology NonLinear Real-Time Expert Seismology seems to be the best tool for on-shore and off-shore petroleum and gas exploration for very deep drillings, ranging up to 20,000 or $30,000 \mathrm{~m}$.
Over the past years, several variants of the integral equations methods were used for the solution of elastodynamic and acoustic problems. It was already at the end of the 1960s when H.A. Shenk ${ }^{1}$ stated that the integral equation for potential mathematically failed to yield unique solutions to the exterior acoustic problem. A method was proposed in which an over-determined system of equations at some characteristic frequencies was formed by combining the surface Helmholtz equation with the corresponding interior Helmholtz equation. It was analytically proved that the system of equations would provide a unique solution at the same characteristic frequencies, to some extent. However, the above method might fail to produce unique solutions, when the interior points used in the collocation of the boundary integral equations are located on a nodal surface of an interior standing wave.

Furthermore, at the beginning of the 1970s Burton and Miller ${ }^{2}$ proposed a combination of the surface Helmholtz integral equation for potential and the integral equation for the normal derivative of potential at the surface, in order to circumvent the problem of nonuniqueness at characteristic frequencies. Their method was named the Composite Helmholtz Integral Equation Method. Some years later, Meyer, Bell, Zinn and Stallybrass, ${ }^{3}$ as well as Terai, ${ }^{4}$ developed regularization techniques for planar elements for the calculation of sound fields around three dimensional objects by integral equation methods.

On the other hand, Reut ${ }^{5}$ further investigated the Composite Helmholtz Integral Equation Method by introducing the hypersingular integrals. Furthermore, in the above numerical method, the accuracy of the integrations affects the results and the conventional Gauss quadrature cannot be used directly. Okada, Rajiyah, and Alturi, ${ }^{6}$ as well as Okada and Alturi $^{9}$ introduced the basic idea of using the gradients of the fundamental solution to the Helmholtz differential equation for velocity potential as vector test functions. This could be completed to write the weak form of the original Helmholtz 